\title{
The new Global Multiple Sclerosis Severity Score (MSSS) correlates with axonal but not glial biomarkers
}

\author{
A. Petzold M.J. Eikelenboom G. Keir \\ C.H. Polman \\ B.M.J. Uitdehaag \\ E.J. Thompson \\ G. Giovannoni \\ 04.08.2005
}

Key words: biomarker, multiple sclerosis, msss

Address correspondence to: Dr Axel Petzold, UCL Institute of Neurology, Department of Neuroinflammation, Queen Square, London WC1N 3BG, United Kingdom.

Tel. +44 (0)2078373611 ext.: 4204, Fax +44 (0)2078378553

Email: a.petzold@ion.ucl.ac.uk 


\begin{abstract}
This study investigated whether the new Global Multiple Sclerosis Severity Scale (MSSS) correlated with cerebrospinal fluid biomarkers for axonal and glial pathology. The MSSS correlated with the phosphorylated neurofilament heavy chain ( $\mathrm{NfH}-\mathrm{SMI} 35, \mathrm{R}=0.44, \mathrm{p}=0.016$ ). The degree of neurofilament phosphorylation (ratio NfH-SMI34 to $\mathrm{NfH}-\mathrm{SMI35}$ ) was 8-fold higher in severely (median MSSS 6.5) versus mildly (MSSS 3.2) disabled patients (7.3 versus $0.9, p=0.03$ ). The MSSS may provide a statistically powerful tool for comparing overall disease severity and be useful for validating the biomarker concept in MS.
\end{abstract}




\section{Introduction}

A new scale based on a statistical approach combining Kurtzke's Expanded Disability Status Scale score (EDSS) with historical databases has been developed. ${ }^{1}$ This new scale, the Global Multiple Sclerosis Severity Score (MSSS) aims to provide a measure for disease severity in an individual patient on a cross-sectional basis. This has been achieved by providing a statistically constructed look-up table of disease severity scores for patients with an EDSS between 0 and 9.5 and a disease duration between 1 to 30 years. This scale is potentially superior to the non-linear EDSS for statistical evaluations, as it combines EDSS and disease duration in one variable that is normally distributed.

Biomarkers for axonal degeneration, such as neurofilaments and glial scar tissue, such as glial fibrillary acidic protein (GFAP) have previously been related to disability and prognosis. ${ }^{2-6}$ There is supporting evidence from animal and cell-culture work that levels of phosphorylated neurofilaments correlate with the loss of axons and function. ${ }^{7-9}$

Here we investigate for the first time (1) whether the newly developed Global MSSS correlates with biomarkers for axonal degeneration (neurofilaments), glial activation (S100B) and astrogliosis (GFAP), and (2) whether these biomarker levels are higher in those patients with more severe disease as defined by the Global MSSS.

\section{Patients and methods}

Data from a previously published cohort of 29 patients with clinically definite MS in whom a 3-year follow-up cerebrospinal fluid (CSF) sample was available ${ }^{10}$ was reanalyzed. The demographic data, representing the 
current disease course, is summarised in the result section. All analyses were based on this follow-up visit.

The phosphorylated neurofilament heavy chain $\left(\mathrm{NfH}^{S M I 35}\right)$, the hyperphosphorylated neurofilament heavy chain $\left(\mathrm{NfH}^{S M I 34}\right)$, glial fibrillary acidic protein (GFAP ${ }^{S M I 26}$ ), S100B and ferritin were quantified as described. ${ }^{3,10}$ The phosphoform ratio $\left(R A T I O=\frac{N f H^{S M I S 4}}{N f H^{S M I S 5}} \times 10\right)$ was used to estimate the degree of phosphorylation, with higher values indicating a higher degree of $\mathrm{NfH}$ phosphorylation. Samples of CSF were obtained by routine lumbar puncture. Aliquots of CSF were stored at $-70^{\circ} \mathrm{C}$ until assayed.

The EDSS was recorded as described, ${ }^{11}$ the progression index was calculated as the ratio of the EDSS to disease duration and the Global MSSS was taken from Figure 3 from the paper by Roxburgh and colleagues. ${ }^{1}$ The progression index was calculated as the EDSS divided by the disease duration.

Data analysis The linear relationship between continuous variables was evaluated using the Spearman correlation coefficient. We were interested testing whether previously reported correlations of biomarkers with disability scales ( $\mathrm{NfH}^{S M I 35}$, GFAP and $\mathrm{S} 100 \mathrm{~B}^{2,3}$ ) would be repeated for the MSSS. The Bonferroni corrected level of significance $(\alpha=0.05)$ for these three comparisons calculates to $p \leq 0.016$. The non-parametric KruskalWallis test was used to compare CSF biomarker levels between patients with mild disease course versus those with a severe disease course according to a previously-used cut-off value of an MSSS of 4.8. ${ }^{12}$ All comparisons were made using SAS software (version 8.2). 


\section{Results}

There were 14 female and 15 male patients with a median age of 46.3 (range 28.5-64.8) years, a median disease duration of 13 (range 3.1-27.6) years and a median relapse-free interval of 24 (range 0.7-270.5) months.

The results for the CSF axonal and glial biomarker levels are summarised in Table 1. Patients with a more severe disease course had an 8 -fold higher $\mathrm{NfH}$ phosphoform ratio (7.3 versus $0.9, p=0.033$ ), indicating a higher degree of $\mathrm{NfH}$ phosphorylation in these patients (Table 1). In contrast, the estimation of disease severity based on the EDSS alone using our previously-published cut-off limit $^{3}$ of 6.5 did not reveal such a difference (data not shown).

The CSF NfH ${ }^{S M I 35}$ levels correlated with the MSSS $(\mathrm{R}=0.44, \mathrm{p}=0.016$, Figure 1). No such correlation was found for either CSF GFAP or CSF S100B levels with the MSSS ( $p=0.81, p=0.39$, respectively).

The Global MSSS correlated with the progression index $(R=0.8, p<0.0001)$. However, when we repeated above analyses using the progression index instead of the Global MSSS, we were not able to show any of above correlations, nor was there a difference between disease severity groups (data now shown).

\section{Discussion}

The finding of a significant, 8-fold increased degree of $\mathrm{NfH}$ phosphorylation, but not of $\mathrm{NfH}^{S M I 35}$ and $\mathrm{NfH}^{S M I 34}$ alone, in patients with a more severe disease course on the Global MSSS is interesting. There is a body of evidence from the dementia and motoneuron literature for an increase of $\mathrm{NfH}$ phosphorylation (reviewed $\mathrm{in}^{2}$ ). Using a different approach we have pre- 
viously demonstrated that $\mathrm{NfH}$ phosphorylation may increase during the progressive phase of MS. ${ }^{10}$ Our previous results were based on the classification of MS patients into those with a relapsing remitting $(R R)$ and those with a secondary or primary progressive (SP/PP) disease course. ${ }^{13}$ The more convincing finding of a 8-fold difference using the $\mathrm{MSSS}^{1}$ versus an about 2-fold difference using the traditional classification ${ }^{13}$ suggests that disease severity and $\mathrm{NfH}$ phosphorylation may be related and independent of the clinical phenotype (i.e. RR versus SP/PP).

The finding that the strength of the correlation between the CSF $\mathrm{NfH}^{S M I 35}$ was marginally better with the EDSS ( $R=0.54)$ [see reference ${ }^{10}$ ] compared to the Global MSSS $(R=0.44)$ in this analysis can be explained by comparing Figure 1 with Figure $2 \mathrm{~A}$ in reference. ${ }^{10}$ One patient with CSF $\mathrm{NfH}^{S M I 35}$ levels of $402 \mathrm{pg} / \mathrm{mL}$, an EDSS of 4 and a disease duration of 21.8 years corresponding to a Global MSSS of 2.53. This patient appears as the outlier in Figure 1. Based on other studies on relapsing MS (reviewed $\mathrm{in}^{2}$ ) one could make the argument that the CSF $\mathrm{NfH}^{S M I 35}$ levels could be related to relapses, but we do not believe this to be the case as the last relapse was 72.9 months ago and CSF neurofilament levels are known to plateau out within 3 months following a relapse. ${ }^{6}$ It is of note that a number of patients with patients with severe disease, i.e. a high MSSS, had non-detectable CSF NfH ${ }^{S M I 35}$ levels. One possible explanation is that axonal degeneration had already occurred a long time ago and biochemical evidence for the damage has been washed out from the CSF. Another possibility is that these patients may have suffered predominantly from cortical damage, which may not necessarily be reflected in lumbar CSF, because of the CSF flow dynamics.

We were not able to demonstrate a correlation between the MSSS and 
the glial biomarkers GFAP and S100B. This finding contrasts with previous reports which showed a correlation between disability scales and CSF GFAP levels. ${ }^{3-5}$ The reason for this is likely to be similar to the arguments

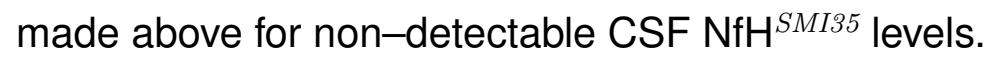

The authors of the Global MSSS highlighted that the difficulty in assessing disability is partly caused by the random damage to more or less relevant anatomical pathways. ${ }^{1}$ Some of this damage will be caused by demyelination and conduction block which may resolve. It seems plausible to assume that the occasional functional recovery, even after a structural deficit (i.e. definite axonal loss) may at least in part be due to central adaption or neuronal plasticity.

Taking this together the Global MSSS appears to be a statistically more powerful tool for comparing overall disease severity than the progression index, and should be evaluated alongside the EDSS in the future studies needed to validate the biomarker concept in MS.

Acknowledgements This study was devised as part of a study into biomarkers for neurodegeneration supported by the Multiple Sclerosis Society of Great Britain and Northern Ireland (AP, GG), the BR Kirk Fund of the Institute of Neurology (AP, ET) and the Multiple Sclerosis Society of the Netherlands (JE).

\section{References}

[1] RHSR Roxburgh, SR Seaman, T Masterman, AE Hensiek, SJ Sawcer, S Vukusic, et al. Multiple Sclerosis Severity Score. Using disability and disease duration to rate disease severity. Neurology, 64:114-1151, 2005. 
[2] A Petzold. Neurofilament phosphoforms: surrogate markers for axonal injury, degeneration \& loss. J Neurol Sci, 233:183-198, 2005.

[3] A Petzold, MJ Eikelenboom, D Gveric, G Keir, M Chapman, RH Lazeron, et al. Markers for different glial cell responses in multiple sclerosis: Clinical and pathological correlations. Brain, 125:1462-1473, 2002.

[4] LE Rosengren, J Lycke, and O Andersen. Glial fibrillary acidic protein in CSF of multiple sclerosis patients - relation to neurological deficit. J Neurol Sci, 133:61-65, 1995.

[5] C Malmestrom, S Haghighi, L Rosengren, O Andersen, and J Lycke. Neurofilament light protein and glial fibrillary acidic protein as biological markers in MS. Neurology, 61:1720-1705, 2003.

[6] JN Lycke, JE Karlsson, O Andersen, and LE Rosengren. Neurofilament protein in cerebrospinal fluid: a potential marker of activity in multiple sclerosis. J Neurol Neurosurg Psychiatry, 64:402-404, 1998.

[7] A Petzold, D Baker, G Pryce, et al. Quantification of neurodegeneration by measurement of brain-specific proteins. J Neuroimmunol, 138:45-48, 2003.

[8] G Pryce, Z Ahmed, DJ Hankey, et al. Cannabinoids inhibit neurodegeneration in models of multiple sclerosis. Brain, 127:2191-2202, 2003.

[9] Samuel J Jackson, David Baker, M. Louise Cuzner, and Lara T Diemel. Cannabinoid-mediated neuroprotection following interferongamma treatment in a three-dimensional mouse brain aggregate cell culture. Eur J Neurosci, 20(9):2267-2275, Nov 2004. 
[10] A Petzold, MJ Eikelenboom, G Keir, et al. Axonal damage accumulates in the progressive phase of multiple sclerosis: A 3-year followup study. J Neurol Neurosurg Psychiatry, 76:206-211, 2005.

[11] JF Kurtzke. Rating neurological impairment in multiple sclerosis: an expanded disability status scale (EDSS). Neurology, 33:1444-1452, 1983.

[12] K. Rejdak, M.J. Eikelenboom, A. Petzold, E.J. Thompson, Z. Stelmasiak, R.H. Lazeron, F. Barkhof, C.H. Polman, B.M. Uitdehaag, and G. Giovannoni. CSF nitric oxide metabolites are associated with activity and progression of multiple sclerosis. Neurology, 63:1439-1445, 2004.

[13] FD Lublin and SC Reingold. Defining the clinical course of multiple sclerosis: results of an international survey National Multiple Sclerosis Society (USA) Advisory Committee on Clinical Trials of New Agents in Multiple Sclerosis. Neurology, 46:907-911, 1996. 
Table 1: Cerebrospinal fluid biomarker levels in MS patients according to disease severity on the Global MSSS. The median (IQR) are shown.

\begin{tabular}{|c|c|c|c|}
\hline & \multirow{2}{*}{$\begin{array}{l}\text { MS } \\
\text { (all) }\end{array}$} & \multicolumn{2}{|c|}{ Disease severity } \\
\hline & & mild & severe \\
\hline MSSS & $4.6(3.1-6.5)$ & $3.1(2.8-4.1)$ & $6.9(5.2-7.5)^{*}$ \\
\hline $\mathrm{NfH}^{S M I 34} \mathrm{pg} / \mathrm{mL}$ & $50(9-129)$ & $17(0-68)$ & $77(30-204)$ \\
\hline $\mathrm{NfH}^{S M I 35} \mathrm{pg} / \mathrm{mL}$ & $113(0-178)$ & $96(0-198)$ & $135(0-223)$ \\
\hline Ratio & $3(1-10.1)$ & $0.9(0.5-3.9)$ & $7.3(2.1-13.4)^{\dagger}$ \\
\hline S100B ng/mL & $0.44(0.35-0.53)$ & $0.44(0.35-0.55)$ & $0.44(0.34-0.51)$ \\
\hline GFAP ${ }^{S M I 26} \mathrm{pg} / \mathrm{mL}$ & $5(4-7)$ & $4(4-7)$ & $5(4-8)$ \\
\hline Ferritin $\mathrm{ng} / \mathrm{mL}$ & $6(5-7)$ & $6(6-7)$ & $6(5-6)$ \\
\hline
\end{tabular}




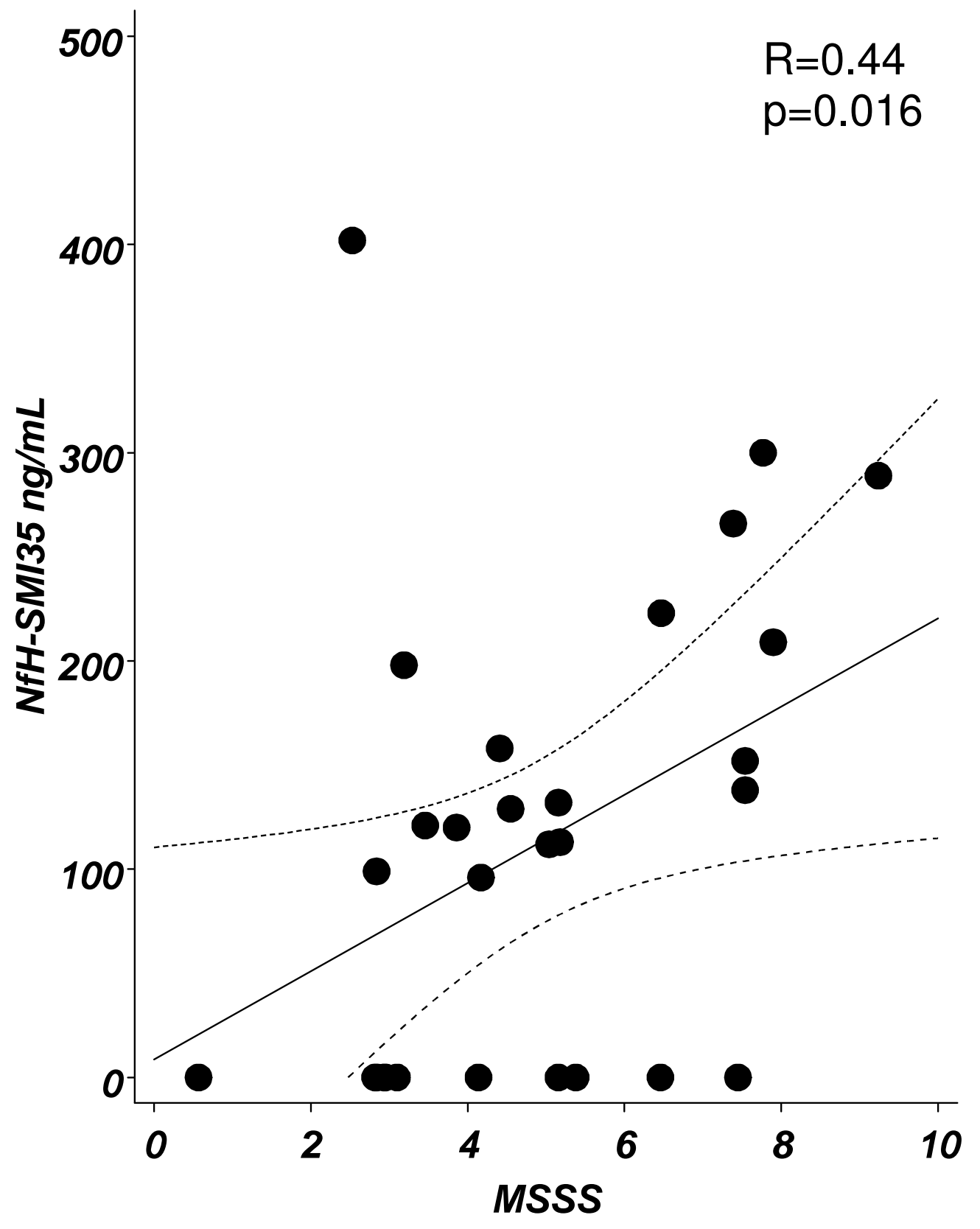

Figure 1: Correlation between the MSSS and CSF NfH ${ }^{S M I 35}$ levels. 